\title{
On the Dynamic Regulation of a Non Linear Model Fish Population
}

\author{
Aboudramane Guiro ${ }^{1}$, Abderrahman $\operatorname{Iggidr}^{2} \&$ Diène Ngom $^{3}$ \\ ${ }^{1}$ Laboratoire d Analyse Mathématique des Equations (LAME), Faculté des Sciences et Techniques, Université de \\ Ouagadougou, Ouagadougou, Burkina Faso \\ 2 EPI MASAIE INRIA Nancy-Grand Est and University Paul Verlaine-Metz LMAM-CNRS UMR 7122, ISGMP \\ Bat. A, Ile du Saulcy 57045 Metz Cedex 01, France \\ ${ }^{3}$ UFR des Sciences et Technologies, Université de Ziguinchor, Ziguinchor, Sénégal \& Laboratoire d Analyse \\ Numérique et d'Informatique Université Gaston Berger de Saint Louis, Sénégal \\ Correspondence: Diène Ngom, UFR des Sciences et Technologies, Université de Ziguinchor, B. P 523 Ziguinchor, \\ Sénégal. E-mail: dngom@univ-zig.sn,ngomdiene@yahoo.fr
}

\author{
Received: February 17, 2013 Accepted: April 16, 2013 Online Published: May 3, 2013 \\ doi:10.5539/jmr.v5n2p84 URL: http://dx.doi.org/10.5539/jmr.v5n2p84
}

\begin{abstract}
In this work we deal with the stability property of a discrete-time nonlinear model of a harvested fish population with the Beverthon-Holt recruitment relationship. We use the method of Lyapunov functions to show that the positive equilibrium state of the stage structured model fish corresponding to a constant fishing intensity is globally and asymptotically stable. Since the fishing intensity is often variable, we also propose how a variable fishing intensity may be controlled through feedback in order to stabilize the fish population state around the positive equilibrium state.
\end{abstract}

Keywords: stage structured population models, discrete-time system, harvested fish population, stability, stabilization

\section{Introduction}

Mathematical models are more and more use for a better management of renewable resources. In the literature we find some mathematical models representing the dynamic evolution of certain renewable resources such as halieutic resources (Doubleday, 1975; Gouze, Rapaport, \& Hadj-Sadok, 2000) and the animal resources (Damania, Phillips, Henson, \& Hayward, 2005; Henson, Hayward, \& Damania, 2005). Using these models and some mathematical tools, we show that it is possible to analyze the dynamical evolution of certain renewable resources and to take decisions which help to protect our natural resources. We are mainly interested in this work to a management problem of halieutic resources using mathematical models (Guiro, Iggidr, \& Ngom, 2008, 2011; Ngom, Iggidr, Guiro, \& Ouahbi, 2008; Ouahbi, 2002). A crucial problem in wild life management policies is how to prevent extinction of some species. This is especially an important problem for depleted fish populations that are subject to heavy fishing efforts. In such cases we have to act on the fish population in order to keep the stock at a desired level, and prevent the extinction of the species. Given that environmental factors are harder to control, the easiest and most practical way to controle the evolution of fish population is by controlling the fishing effort. Here we analyze a mathematical model (1) of fish populations and show that with a constant fishing effort the non trivial positive equilibrium state of the model is globally asymptotically stable. Generally those fishing efforts are variables, so we analyze how these variations could affect the stability of the fish population. We show how to stabilize the system around the steady state by using a feedback mechanism that is dependent on the fish population state to control the variation of the fishing effort. The stability property we ensure here has a fundamental biological consequence. It ensures that, if the model is valid, the stock of fish population will never turn to extinction. A similar problem has been considered in (Bagdouri, Iggidr, Ouahbi, \& Vivalda, 2000) for an age-structured model, but we think that it is more practical to structure a fish population according to its stage of growth such as the weight or the length. For this end, we consider the following discrete time system which describe the dynamic evolution of a haversted fish population (see for instance Getz and Haight (1989) for more detail in the synthesis of the model): 


$$
\left\{\begin{aligned}
X_{1}(k+1) & =\left(1-p_{1}\right) e^{\left(-M_{1}-q_{1} E(k) \tau\right)} X_{1}(k)+s_{0}\left(\sum_{i=1}^{n} b_{i} X_{i}(k)\right) h\left(\sum_{i=1}^{n} b_{i} X_{i}(k)\right) \\
X_{2}(k+1) & =p_{1} e^{\left(-M_{1}-q_{1} E(k) \tau\right)} X_{1}(k)+\left(1-p_{2}\right) e^{\left(-M_{2}-q_{2} E(k) \tau\right)} X_{2}(k) \\
& \vdots \\
X_{n-1}(k+1) & =p_{n-2} e^{\left(-M_{n-2}-q_{n-2} E(k) \tau\right)} X_{n-2}(k)+\left(1-p_{n-1}\right) e^{\left(-M_{n-1}-q_{n-1} E(k) \tau\right)} X_{n-1}(k) \\
X_{n}(k+1) & =p_{n-1} e^{\left(-M_{n-1}-q_{n-1} E(k) \tau\right)} X_{n-1}(k)+e^{\left(-M_{n}-q_{n} E(k) \tau\right)} X_{n}(k)
\end{aligned}\right.
$$

where:

- $n$ is the total stage-classes number.

- $X_{i}(k)$ is the population size in the stage class $i$ at time $k$.

- $b_{i}$ is the fecundity rate of class $i$.

- $M_{i}$ is the natural death rate in the class $i$.

- $q_{i}$ is the individual catchability coefficient in the class $i$.

- $E(k)$ is the fishing intensity at time $k$.

- $p_{i}$ is the transition rate of individuals in stage class $i$ to the stage class $i+1$ after the time interval $[k, k+1]$.

- $\tau$ is the time periode where fishing activity is authorized.

- $h$ is the Beverthon-Holt recruitment relationship.

The Beverthon-Holt recruitment relationship $h$ is in the form:

$$
h(x)=\frac{1}{1+\beta x}, \text { where } \beta \text { is a positive reel number }
$$

Generally, recruitment relationship are decreasing continuous function on $\mathbb{R}^{+}$on to [0,1]. Many mathematical expressions have been proposed for the stock-recruitment relationship in the literature (Beverton, \& Holt, 1957; Getz, \& Haight, 1989; Magal, \& Pelletier, 1997; Ricker, 1954; Shepherd, 1982).

To simplify the notation, we note Equation (1) by:

$$
X(k+1)=F(X(k), E(k))=\left(\begin{array}{c}
F_{1}(X(k), E(k)) \\
F_{2}(X(k), E(k)) \\
\vdots \\
F_{n}(X(k), E(k))
\end{array}\right)
$$

where:

$$
\begin{gathered}
F_{1}(X(k), E(k))=\left(1-p_{1}\right) s_{1}(k) X_{1}(k)+s_{0}\left(\sum_{i=1}^{n} b_{i} X_{i}(k)\right) h\left(\sum_{i=1}^{n} b_{i} X_{i}(k)\right) \\
F_{i}(X(k), E(k))=p_{i-1} s_{i-1}(k) X_{i-1}(k)+\left(1-p_{i}\right) s_{i}(k) X_{i}(k) \text { for } i=2, \ldots, n-1 \\
F_{n}(X(k), E(k))=p_{n-1} s_{n-1}(k) X_{n-1}(k)+s_{n}(k) X_{n}(k) \\
s_{i}(k)=e^{\left(-M_{i}-q_{i} E(k) \tau\right)}, \forall i=1, \ldots, n \text { and } \forall k \geq 0 .
\end{gathered}
$$

Now we briefly recall some mathematical definitions and results on discrete time stability theory that will be use later in this work.

Definition 1 The system (2) is stable (or Lyapunov stable) at $X^{*}$ if for each $\epsilon$ there exists a positive real number $\delta$ such that for each initial state $X_{0}$ with $\left|X_{0}-X^{*}\right|<\delta$, the solution $X(k)$ of system (2) (with the initial condition $X_{0}$ ) is defined for all $k>0$ and satisfies $\left|X(k)-X^{*}\right|<\epsilon$ for all $k>0$.

Definition 2 The equilibrium state $X^{*}$ of system (2) is said to be attractive if there exists a neighborhood $U$ of $X^{*}$ such that for any initial condition $X_{0}$ belonging to $U$ the corresponding solution $X(k)$ is defined for all $k \geq 0$ and tends to $X^{*}$ as $k$ tends to infinity. If $U$ is the whole state space then the equilibrium state $X^{*}$ is globally attractive. 
Definition 3 The system (2) is asymptotically stable at $X^{*}$ if it is stable and attractive at $X^{*}$.

More details about the above concept can be found in (Elaydi, 1996; Iggidr, 2006; Sontag, 1998).

\section{Stability Analysis of the Model}

\subsection{Equilibrium State}

Let us consider a constant fishing intensity $E(k)=E^{*}$ and denote $X^{*}=\left(X_{1}^{*}, X_{2}^{*}, \ldots, X_{n}^{*}\right)$ the corresponding positive equilibrium state of model (1); then $X^{*}$ must satisfy:

$$
\left\{\begin{aligned}
X_{1}^{*} & =\left(1-p_{1}\right) s_{1} X_{1}^{*}+s_{0}\left(\sum_{i=1}^{n} b_{i} X_{i}^{*}\right) h\left(\sum_{i=1}^{n} b_{i} X_{i}^{*}\right) \\
X_{2}^{*} & =p_{1} s_{1} X_{1}^{*}+\left(1-p_{2}\right) s_{2} X_{2}^{*} \\
& \vdots \\
X_{n-1}^{*} & =p_{n-2} s_{n-2} X_{n-2}^{*}+\left(1-p_{n-1}\right) s_{n-1} X_{n-1}^{*} \\
X_{n}^{*} & =p_{n-1} s_{n-1} X_{n-1}^{*}+s_{n} X_{n}^{*}
\end{aligned}\right.
$$

System (3) can be written as:

$$
\left\{\begin{aligned}
X_{1}^{*} & =\frac{s_{0}}{1-\left(1-p_{1}\right) s_{1}}\left(\sum_{i=1}^{n} b_{i} X_{i}^{*}\right) h\left(\sum_{i=1}^{n} b_{i} X_{i}^{*}\right) \\
X_{2}^{*} & =\frac{p_{1} s_{1}}{1-\left(1-p_{2}\right) s_{2}} X_{1}^{*} \\
& \vdots \\
X_{n-1}^{*} & =\frac{p_{n-2} s_{n-2}}{1-\left(1-p_{n-1} s_{n-1}\right)} X_{n-2}^{*} \\
X_{n}^{*} & =\frac{p_{n-1} s_{n-1}}{1-s_{n}} X_{n-1}^{*}
\end{aligned}\right.
$$

Let us put:

$$
l_{1}=1, \quad l_{j}=\prod_{i=2}^{j} \frac{p_{i-1} s_{i-1}}{1-\left(1-p_{i}\right) s_{i}}, j=2, \ldots, n-1 \text { and } l_{n}=\frac{p_{n-1} s_{n-1}}{1-s_{n}} \prod_{i=2}^{n-1} \frac{p_{i-1} s_{i-1}}{1-\left(1-p_{i}\right) s_{i}}
$$

Using relations between $X_{i}^{*}$, we notice that:

$$
X_{i}^{*}=l_{i} X_{1}^{*} \quad i=2, \ldots n
$$

Let us define (as it has been done in Getz \& Haight, 1989, p. 25) $R=\sum_{i=1}^{n} b_{i} l_{i}$, then we can write:

$$
\sum_{i=1}^{n} b_{i} X_{i}^{*}=\left(b_{1} l_{1}+b_{2} l_{2}+\ldots+b_{n} l_{n}\right) X_{1}^{*}=R X_{1}^{*}
$$

Following the first equation of system (4), we show that:

$$
X_{1}^{*}=\frac{1}{R} h^{-1}\left(\frac{1-\left(1-p_{1}\right) s_{1}}{s_{0} R}\right)=\frac{1}{\beta R} \frac{s_{0} R-\left(1-\left(1-p_{1}\right) s_{1}\right)}{1-\left(1-p_{1}\right) s_{1}}
$$

Remark 1 The steady point $X^{*}$ has biological meaning (positivity of the steady state) if and only if $R>\frac{1-\left(1-p_{1}\right) s_{1}}{s_{0}}$. We will now assume that the latter inequality is satisfied.

\subsection{Main Results}

\subsubsection{Stability Analysis of the Equilibrium Point $X^{*}$}

Let considere the notations in the previous section, our fisrt main result is the stability property: 
Theorem 1 Let's consider that the fishing intensity is constant, that is $E(k)=E^{*} \forall k \geq 0$, then the positive equilibrium state $X^{*}$ of model (1) is globally asymptotically stable.

For the proof of Theorem 1 we shall use the following results:

2nd Lyapunov Theorem Suppose there exists a $C^{1}$ function $V$ defined on some neighborhood $U$ of the equilibrium state $X^{*}$ such that:

i) $V(X)>0$ for all $X \in U \backslash\{0\}$ and $V\left(X^{*}\right)=0$.

ii) $V\left(F\left(X, E^{*}\right)\right)-V(X) \leq 0$ for all $X \in U$.

Then the system (2) is Lyapunov stable at equilibrium state $X^{*}$.

Lasalle's Invariance Principle Let $\Omega$ be a subset of $\mathbb{R}^{n}$. Assume that $\Omega$ is positively invariant for system (2). Let $V: \Omega \rightarrow \mathbb{R}$ be a $C^{1}$ function scalar such that $V\left(F\left(X, E^{*}\right)\right)-V(X) \leq 0$ for all $k \in \mathbb{N}$ in $\Omega$. Let $\mathcal{E}$ be the set of points within $\Omega$ where $V\left(F\left(X, E^{*}\right)\right)-V(X)=0$ and let $\mathcal{L}$ the largest invariant set within $\mathcal{E}$. Then every bounded solution starting in $\Omega$ tends to the set $\mathcal{L}$ as $k$ goes to infinity.

For the proof of the 2nd Lyapunov Theorem and the Lasalle's invariance principle, one can see for instance (Hurt, 1967; Kalman, \& Bertram, 1960; Lasalle, 1986).

Proof of Theorem 1. Using the notations in section 2.1, let's define: $R_{i}=\frac{b_{i} l_{i}}{R}$ for $i=1, \ldots, n$ and

$$
V(X)=\left(X_{1}-X_{1}^{*}\right)^{2}+\sum_{i=2}^{n-1}\left[\frac{1-\left(1-p_{1}\right) s_{1}}{1-\left(1-p_{i}\right) s_{i}} \sum_{j=i}^{n} R_{j}\right]\left(\frac{X_{i}-X_{i}^{*}}{l_{i}}\right)^{2}+\frac{\left(1-\left(1-p_{1}\right) s_{1}\right) R_{n}}{1-s_{n}}\left(\frac{X_{n}-X_{n}^{*}}{l_{n}}\right)^{2}
$$

We verify that $V\left(X^{*}\right)=0$ and $V(X) \geq 0 \forall X \in \mathbb{R}^{n}$

Let us compute the variation of $V$ along the solutions of system (2).

$$
\begin{aligned}
V(X(k+1)) & =\left(X_{1}(k+1)-X_{1}^{*}\right)^{2}+\sum_{i=2}^{n-1}\left[\frac{1-\left(1-p_{1}\right) s_{1}}{1-\left(1-p_{i}\right) s_{i}} \sum_{j=i}^{n} R_{j}\right]\left(\frac{X_{i}(k+1)-X_{i}^{*}}{l_{i}}\right)^{2} \\
& +\frac{\left(1-\left(1-p_{1}\right) s_{1}\right) R_{n}}{1-s_{n}}\left(\frac{X_{n}(k+1)-X_{n}^{*}}{l_{n}}\right)^{2}
\end{aligned}
$$

using the dynamics of system (2), we obtain:

$$
\begin{aligned}
V(X(k+1)) & =\left(\left(1-p_{1}\right) s_{1}\left(X_{1}(k)-X_{1}^{*}\right)+\frac{s_{0} \sum_{i=1}^{n} b_{i}\left(X_{i}(k)-X_{i}^{*}\right)}{\left(1+\beta \sum_{i=1}^{n} b_{i} X_{i}(k)\right)\left(1+\beta \sum_{i=1}^{n} b_{i} X_{i}^{*}\right)}\right)^{2} \\
& +\sum_{i=2}^{n-1}\left[\frac{1-\left(1-p_{1}\right) s_{1}}{1-\left(1-p_{i}\right) s_{i}} \sum_{j=i}^{n} R_{j}\right]\left(\frac{p_{i-1} s_{i-1}\left(X_{i-1}(k)-X_{i-1} *\right)+\left(1-p_{i}\right) s_{i}\left(X_{i}(k)-X_{i}^{*}\right)}{l_{i}}\right)^{2} \\
& +\frac{\left(1-\left(1-p_{1}\right) s_{1}\right) R_{n}}{1-s_{n}}\left(\frac{p_{n-1} s_{n-1}\left(X_{n-1}(k)-X_{n-1}^{*}\right)+s_{n}\left(X_{n}(k)-X_{n}^{*}\right)}{l_{n}}\right)^{2}
\end{aligned}
$$

Since $X_{i}^{*}=l_{i} X_{1}^{*}, i=2, \ldots n$, we show that $1+\beta \sum_{i=1}^{n} b_{i} X_{i}^{*}=\frac{s_{0} R}{1-\left(1-p_{1}\right) s_{1}}$, hence:

$$
\begin{aligned}
V(X(k+1)) & =\left(\left(1-p_{1}\right) s_{1}\left(X_{1}(k)-X_{1}^{*}\right)+\frac{1-\left(1-p_{1}\right) s_{1}}{1+\beta \sum_{i=1}^{n} X_{i}(k)} \sum_{i=1}^{n} \frac{b_{i} l_{i}}{R}\left(\frac{X_{i}(k)-X_{i}^{*}}{l_{i}}\right)\right)^{2} \\
& +\sum_{i=2}^{n-1}\left[\frac{1-\left(1-p_{1}\right) s_{1}}{1-\left(1-p_{i}\right) s_{i}} \sum_{j=i}^{n} R_{j}\right]\left(\left(1-\left(1-p_{i}\right) s_{i}\right)\left(\frac{X_{i-1}(k)-X_{i-1} *}{l_{i-1}}\right)+\left(1-p_{i}\right) s_{i}\left(\frac{X_{i}(k)-X_{i}^{*}}{l_{i}}\right)^{2}\right. \\
& +\left(1-\left(1-p_{1}\right) s_{1}\right) \frac{R_{n}}{1-s_{n}}\left(\left(1-s_{n}\right)\left(\frac{X_{n-1}(k)-X_{n-1}^{*}}{l_{n-1}}\right)+s_{n}\left(\frac{X_{n}(k)-X_{n}^{*}}{l_{n}}\right)\right)^{2}
\end{aligned}
$$


Since the map: $X \mapsto X^{2}$ is convexe, we have the inequality:

$$
\begin{aligned}
V(X(k+1)) \leq & \left(1-p_{1}\right) s_{1}\left(X_{1}(k)-X_{1}^{*}\right)^{2}+\left(1-\left(1-p_{1}\right) s_{1}\right)\left(\frac{1}{1+\beta \sum_{i=1}^{n} X_{i}(k)} \sum_{i=1}^{n} \frac{b_{i} l_{i}}{R}\left(\frac{X_{i}(k)-X_{i}^{*}}{l_{i}}\right)\right)^{2} \\
& +\sum_{i=2}^{n-1}\left[\frac{1-\left(1-p_{1}\right) s_{1}}{1-\left(1-p_{i}\right) s_{i}} \sum_{j=i}^{n} R_{j}\right]\left[\left(1-\left(1-p_{i}\right) s_{i}\right)\left(\frac{X_{i-1}(k)-X_{i-1} *}{l_{i-1}}\right)^{2}+\left(1-p_{i}\right) s_{i}\left(\frac{X_{i}(k)-X_{i}^{*}}{l_{i}}\right)^{2}\right] \\
& +\left(1-\left(1-p_{1}\right) s_{1}\right) \frac{R_{n}}{1-s_{n}}\left[\left(1-s_{n}\right)\left(\frac{X_{n-1}(k)-X_{n-1}^{*}}{l_{n-1}}\right)^{2}+s_{n}\left(\frac{X_{n}(k)-X_{n}^{*}}{l_{n}}\right)^{2}\right]
\end{aligned}
$$

Otherwise $1+\beta \sum_{i=1}^{n} X_{i}(k) \geq 1$ and $\frac{b_{i} l_{i}}{R}=R_{i}$ imply:

$$
\begin{aligned}
V(X(k+1)) & \leq\left[1-\left(1-\left(1-p_{1}\right) s_{1}\right) R_{1}\right]\left(X_{1}(k)-X_{1}^{*}\right)^{2}+\left(1-\left(1-p_{1}\right) s_{1}\right)\left(\sum_{i=1}^{n} R_{i}\left(\frac{X_{i}(k)-X_{i}^{*}}{l_{i}}\right)\right)^{2} \\
& +\sum_{i=2}^{n-1}\left[\frac{1-\left(1-p_{1}\right) s_{1}}{1-\left(1-p_{i}\right) s_{i}} \sum_{j=i}^{n} R_{j}-\left(1-\left(1-p_{1}\right) s_{1}\right) R_{i}\right]\left(\frac{X_{i}(k)-X_{i}^{*}}{l_{i}}\right)^{2} \\
& +\left[-\left(1-\left(1-p_{1}\right) s_{1}\right) R_{n}+\left(1-\left(1-p_{1}\right) s_{1}\right) \frac{R_{n}}{1-s_{n}}\right]\left(\frac{X_{n}(k)-X_{n}^{*}}{l_{n}}\right)^{2}
\end{aligned}
$$

hence,

$$
\begin{aligned}
& V(X(k+1))-V(X(k)) \leq\left(1-\left(1-p_{1}\right) s_{1}\right)\left(\sum_{i=1}^{n} \frac{R_{i}\left(X_{i}(k)-X_{i}^{*}\right)}{l_{i}}\right)^{2}\left(1-\left(1-p_{1}\right) s_{1}\right) R_{1}\left(X_{1}(k)-X_{1}^{*}\right)^{2} \\
&-\sum_{i=2}^{n-1}\left(1-\left(1-p_{1}\right) s_{1}\right) R_{i}\left(\frac{X_{i}(k)-X_{i}^{*}}{l_{i}}\right)^{2}-\left(1-\left(1-p_{1}\right) s_{1}\right) R_{n}\left(\frac{X_{n}(k)-X_{n}^{*}}{l_{n}}\right)^{2} \\
& V(X(k+1))-V(X(k)) \leq\left(1-\left(1-p_{1}\right) s_{1}\right)\left[\left(\sum_{i=1}^{n} R_{i}\left(\frac{X_{i}(k)-X_{i}^{*}}{l_{i}}\right)\right)^{2}-\sum_{i=1}^{n} R_{i}\left(\frac{X_{i}(k)-X_{i}^{*}}{l_{i}}\right)^{2}\right]
\end{aligned}
$$

Therefore $V(X(k+1))-V(X(k)) \leq 0$. Hence, by the 2 nd Lyapunov Theorem, we can say that the equilibrium state $X^{*}$ of system (2) is stable.

Now let us define:

$$
\mathcal{E}=\left\{X \in \mathbb{R}_{+}^{n} / V\left(F\left(X, E^{*}\right)\right)-V(X)=0\right\}
$$

and let $\mathcal{L}$ be the largest invariant set within $\mathcal{E}$. Let $X$ in $\mathcal{L}$, then using (6) we can write:

$$
\left(\sum_{i=1}^{n} R_{i}\left(\frac{X_{i}-X_{i}^{*}}{l_{i}}\right)\right)^{2}=\sum_{i=1}^{n} R_{i}\left(\frac{X_{i}-X_{i}^{*}}{l_{i}}\right)^{2}
$$

While $\sum_{i=1}^{n} R_{i}=1$, by strict convexity of the map $X \mapsto X^{2}$, we obtain: $\frac{X_{i}-X_{i}^{*}}{l_{i}}=X_{1}-X_{1}^{*}$, for $i=1, \ldots, n$.

Therefore $X_{i}=l_{i} X_{1}$.

Otherwise

$$
\begin{aligned}
V\left(F\left(X, E^{*}\right)\right)-V(X) & =\left(F_{1}\left(X, E^{*}\right)-X_{1}^{*}\right)^{2}+\sum_{i=2}^{n-1}\left[\frac{1-\left(1-p_{1}\right) s_{1}}{1-\left(1-p_{i}\right) s_{i}} \sum_{j=i}^{n} R_{j}\right]\left(\frac{F_{i}\left(X, E^{*}\right)-X_{i}^{*}}{l_{i}}\right)^{2} \\
& +\left(1-\left(1-p_{1}\right) s_{1}\right) \frac{R_{n}}{1-s_{n}}\left(\frac{F_{n}\left(X, E^{*}\right)-X_{n}^{*}}{l_{n}}\right)^{2}-\left(X_{1}-X_{1}^{*}\right)^{2} \\
& -\sum_{i=2}^{n-1}\left[\frac{1-\left(1-p_{1}\right) s_{1}}{1-\left(1-p_{i}\right) s_{i}} \sum_{j=i}^{n} R_{j}\right]\left(\frac{X_{i}-X_{i}^{*}}{l_{i}}\right)^{2}\left(1-\left(1-p_{1}\right) s_{1}\right) \frac{R_{n}}{1-s_{n}}\left(\frac{X_{n}-X_{n}^{*}}{l_{n}}\right)^{2}
\end{aligned}
$$


Using Equation (2) we show that $F_{i}\left(X, E^{*}\right)=X_{i}$ for $i=2, \ldots, n$. Hence $V\left(F\left(X, E^{*}\right)\right)-V(X)=\left(F_{1}\left(X, E^{*}\right)-X_{1}^{*}\right)^{2}-$ $\left(X_{1}-X_{1}^{*}\right)^{2}$. Since $X \in \mathcal{L} \subset \mathcal{E}$ then $\left(F_{1}\left(X, E^{*}\right)-X_{1}^{*}\right)^{2}-\left(X_{1}-X_{1}^{*}\right)^{2}=0$, that is $F_{1}\left(X, E^{*}\right)=X_{1}$. This show that $F\left(X, E^{*}\right)=X$ if $X \in \mathcal{L}$, therefore $\mathcal{L} \subseteq\left\{0, X^{*}\right\}$.

Now we show that $0 \notin \mathcal{L}$. Assume that $0 \in \mathcal{L}$. Hence there exist $X^{0}$ in $\mathbb{R}_{+}^{n}$ such that the solution $X(k)$ which starts from $X^{0}$ tends to zero when $k$ tends to infinity.

$V$ is a decreasing function along the solutions, then:

$$
V\left(X^{0}\right) \geq V(X(k)) \geq V(0) \forall k \geq 0 .
$$

But

$$
\begin{gathered}
V(0)-V\left(X^{0}\right)=X_{1}^{* 2}+\sum_{i=2}^{n-1}\left[\frac{1-\left(1-p_{1}\right) s_{1}}{1-\left(1-p_{i}\right) s_{i}} \sum_{j=i}^{n} R_{j}\right]\left(\frac{X_{i}^{*}}{l_{i}}\right)^{2}+\left(1-\left(1-p_{1}\right) s_{1}\right) \frac{R_{n}}{1-s_{n}}\left(\frac{X_{n}^{*}}{l_{n}}\right)^{2} \\
-\left(X_{1}^{0}-X_{1}^{*}\right)^{2}-\sum_{i=2}^{n-1}\left[\frac{1-\left(1-p_{1}\right) s_{1}}{1-\left(1-p_{i}\right) s_{i}} \sum_{j=i}^{n} R_{j}\right]\left(\frac{X_{i}^{0}-X_{i}^{*}}{l_{i}}\right)^{2}-\left(1-\left(1-p_{1}\right) s_{1}\right) \frac{R_{n}}{1-s_{n}}\left(\frac{X_{n}^{0}-X_{n}^{*}}{l_{n}}\right)^{2} \\
V(0)-V\left(X^{0}\right)=X_{1}^{0}\left(2 X_{1}^{*}-X_{1}^{0}\right)^{2}+\sum_{i=2}^{n-1}\left[\frac{1-\left(1-p_{1}\right) s_{1}}{1-\left(1-p_{i}\right) s_{i}} \sum_{j=i}^{n} R_{j}\right]\left(\frac{X_{i}^{0}}{l_{i}}\right)\left(\frac{2 X_{i}^{*}-X_{i}^{0}}{l_{i}}\right)^{2}+\left(1-\left(1-p_{1}\right) s_{1}\right) \frac{R_{n}}{1-s_{n}}\left(\frac{X_{n}^{0}}{l_{n}}\right)\left(\frac{2 X_{n}^{*}-X_{n}^{0}}{l_{n}}\right)^{2}
\end{gathered}
$$

$V(0)>V(X)$ for all $x \in\left\{X \in \mathbb{R}_{+}^{n}: 0<X_{i}<2 X_{i}^{*}, \forall i=1, \ldots, n\right\}$, this is contradiction with relation (7). Hence $\mathcal{L}=\left\{X^{*}\right\}$. The steady point $X^{*}$ is then globally asymptotically stable thanks to Lasalle's invariance principle.

\subsubsection{Feedback Stabilization}

We established in the previous proposition that for a constant fishing intensity, the positive equilibrium state of the model (1) is globally asymptotically stable. However in fishery management the fishing intensity is very often a parameter which varies according to the abundance of the stocks. Now we focus our attention in the problem which consist in stabilizing the state of the fish population around the equilibrium state by acting on the fishing intensity which we suppose variable in function of the fish population state. In control theory there are two ways to stabilize a system around an equilibrium state. The first one consists in finding the control as a function of only the time variable $k$ and corresponds to what is called an open-loop control. The second strategy is to build the stabilizing control as a function of the state $X(k)$ of the population, this is a feedback control or a closed-loop control. Here we develop the basis of the second strategy because as we mentioned it previously, the fishing intensity often depends on the abundance of the stocks fish population.

The considered system is nonlinear so the classical linear control techniques can not be used (unless to get local results but here we are interested in global stablization by means of a nonegative feedback control). Thus, we shall use a discrete version of Jurdjevic-Quinn theorem (Jurdjevic, \& Quinn, 1978) that has been derived in (Bensoubaya, Ferfera, \& Iggidr, 1995; Bensoubaya, Ferfera, \& Iggidr, 1995; Lin, 1996). Let $E^{*}$ be a given constant value of the fishing intensity and $x^{*}$ the corresponding positive equilibrium state. To construct the stabilizing feedback, we simply use the same method that has been used in (Bagdouri, Iggidr, Ouahbi, \& Vivalda, 2000). To be self contained, we recall here this result as well as its proof.

Theorem 2 Let $\eta$ such that $0<\eta \leq E^{*}$, then the system (1) is globally stabilizable by the feedback $E(X)=E^{*}+v(X)$, where $v(X)$ is continuous and satisfy $\|v(X)\| \leq \eta, \quad \forall X \in \mathbb{R}^{+}$.

Proof Theorem 2. We consider the Lyapunov function $V$ defined in (5).

$$
\Delta V(X)=V\left(F(X, E(X))-V(X)=V\left(F\left(X, E^{*}+v(X)\right)-V(X)\right.\right.
$$

The Taylor expansion at the point $\left(x, E^{*}\right)$ gives

$$
\begin{gathered}
\Delta V(X)=V\left(F\left(X, E^{*}\right)+v(X) \frac{\partial}{\partial E} V\left(F\left(X, E^{*}\right)\right)+v(X)^{2} \int_{0}^{1}(1-t) \frac{\partial^{2}}{\partial E^{2}} V\left(F\left(X, E^{*}+t v(X)\right)\right) d t-V(X)\right. \\
\Delta V(X)=V\left(F\left(X, E^{*}\right)-V(X)+v(X) \frac{\partial V}{\partial X}\left(F\left(X, E^{*}\right)\right) \frac{\partial F}{\partial E}\left(X, E^{*}\right)+v(X)^{2} \int_{0}^{1}(1-t) \frac{\partial^{2}}{\partial E^{2}} V\left(F\left(X, E^{*}+t v(X)\right)\right) d t\right.
\end{gathered}
$$


Let $\phi: \Omega \times R \longrightarrow R$ define by

$$
\phi(X, v)=\int_{0}^{1}(1-t) \frac{\partial^{2}}{\partial E^{2}} V\left(F\left(X, E^{*}+t v(X)\right)\right) d t
$$

For $\eta>0$, let $\theta_{1}$ and $\theta_{2}$ be two continuous positive real functions such that $\theta_{1}(X)+\theta_{2}(X) \neq 0, \forall X \in \Omega$ and

$$
\begin{gathered}
\theta_{1}(X)>\sup _{|v| \leq \eta}|\phi(X)|, \forall X \in \Omega \\
\theta_{2}(X)>\left|\frac{\partial V}{\partial X}\left(F\left(X, E^{*}\right)\right) \frac{\partial F}{\partial E}\left(X, E^{*}\right)\right|, \forall X \in \Omega
\end{gathered}
$$

Let define:

$$
\theta(X)=\frac{\eta}{\eta \theta_{1}(X)+\theta_{2}(X)}>0 \forall X \in \Omega
$$

We construct the feedback $v(X)$ by:

$$
v(X)=-\theta(X)\left(\frac{\partial V}{\partial X}\left(F\left(X, E^{*}\right)\right) \frac{\partial F}{\partial E}\left(X, E^{*}\right)\right)
$$

We easily verify that

$$
|v(X)| \leq \eta, \forall X \in \Omega
$$

And we have:

$$
\Delta V(X)=V\left(F\left(X, E^{*}\right)-V(X)-v^{2}(X)\left(\frac{1}{\theta(X)}-\phi(X)\right) .\right.
$$

It's has been show in the proof of Proposition 1 that $V\left(F\left(X, E^{*}\right)-V(X) \leq 0\right.$.

By relations (8), (9) and (10) we verify that $\frac{1}{\theta(X)}-\phi(X) \geq \frac{\left.\theta_{(} X\right)}{\eta} \geq 0$. Hence $\Delta V(X) \leq 0$, that is $X^{*}$ is Lyapunov stable for the feedback $E(X)=E^{*}+v(X)$.

Now we show that $X^{*}$ is attractive. For this end, let $\mathcal{L}_{0}$ be the largest invariant set contained in $\mathcal{E}_{0}=\{X \in$ $\left.\mathbb{R}^{+} / \triangle V(X)=0\right\}$.

Let $x$ in $\mathcal{L}_{0}$, then we have $\Delta V(X)=0$. Since $V\left(F\left(x, E^{*}\right)\right)-V(X) \leq 0$ and $-v^{2}(X)\left(\frac{1}{\theta(X)}-\phi(X)\right) \leq 0$ then $V\left(F\left(x, E^{*}\right)\right)-V(X)=0$ and $v(X)=0$. This shows that $\mathcal{L}_{0} \subset \mathcal{L}$ where $\mathcal{L}$ is the Lasalle's set definded in the proof of Theorem 1 and we have already shown that $\mathcal{L}=\left\{x^{*}\right\}$. We conclude that $\mathcal{L}_{0}=\left\{X^{*}\right\}$, and hence $X^{*}$ is globally attractive by Lasalle's invariance principle. Finally $X^{*}$ is globally asymptotically stable with the feedback $E(X)=E^{*}+v(X)$.

\subsubsection{Discussion}

In this model of fish population (1), we consider the fishing intensity $E(k)$ as a control and we established two stability results by making hypotheses on the fishing intensity. The fishing intensity is a parameter estimated according to all the factors used during the fishing activity. Among these factors we can cite for example the fishing time, the sizes of fishnets, the navigation capacity of the used boats. At first we have established in Theorem 1 that the positive equilibrium state corresponding to a constant fishing intensity is globally asymptotically stable. The result is interesting in the sense that when we suppose that we can maintain at a constant level the fishing intensity on a given fish population whose state dynamic evolution is described by the model (1), we are insured that the stock fish population is stabilized around the positive equilibrium state. In a biological sense, the Theorem 1 means that a constant fishing intensity on a given fish population shall avoid the extinction of this fish population. Secondly taking into account the fact that in practice the fishing intensity is variable, we show in Theorem 2 the we can compute the fishing intensity as a function of stock such that the positive equilibrium state remain globally asymptotically stable. So we have here an example which shows how mathematical tools can be useful for a good management of renewable resources. 


\section{Numerical Exemple}

To illustrate the efficiency of the stabilization method, we give some simulations of the dynamics evolution of the stock fish population. We assume that $n=3$ in model (1).

$$
\left\{\begin{array}{l}
X_{1}(k+1)=\left(1-p_{1}\right) e^{\left(-M_{1}-q_{1} E(k) \tau\right)} X_{1}(k)+s_{0} \frac{\sum_{i=1}^{3} b_{i} X_{i}(k)}{1+\beta\left(\sum_{i=1}^{3} b_{i} X_{i}(k)\right)} \\
X_{2}(k+1)=p_{1} e^{\left(-M_{1}-q_{1} E(k) \tau\right)} X_{1}(k)+\left(1-p_{2}\right) e^{\left(-M_{2}-q_{2} E(k) \tau\right)} X_{2}(k), \\
X_{3}(k+1)=p_{2} e^{\left(-M_{2}-q_{2} E(k) \tau\right)}(k) X_{2}(k)+e^{\left(-M_{3}-q_{3} E(k) \tau\right)} X_{3}(k) .
\end{array}\right.
$$

We assume that the biological parameters in the model (1) are given by:

Parameters of Beverthon-Holt recruitment relationship

Fecundity rates

Individual catchability coefficients

Natural death rate

the transition rate of individuals in stage class $i$ to the stage class $i+1$

Duration of the time harvest season

Constante fishing intensity

Hypothesis theorem 2: $0<\eta \leq E^{*}$

$$
\begin{aligned}
& s_{0}=0.6, \beta=0.0002 \text {, } \\
& b=\left[\begin{array}{lll}
15 & 20 & 20
\end{array}\right] \text {, } \\
& q=\left[\begin{array}{lll}
0.24 & 0.36 & 0.42
\end{array}\right], \\
& M=\left[\begin{array}{lll}
0.2 & 0.2 & 0.2
\end{array}\right], \\
& p_{1}=0.5, p_{2}=0.7, p_{3}=0 \\
& \tau=2 / 3 \text {, } \\
& E^{*}=8 \\
& \eta=6
\end{aligned}
$$

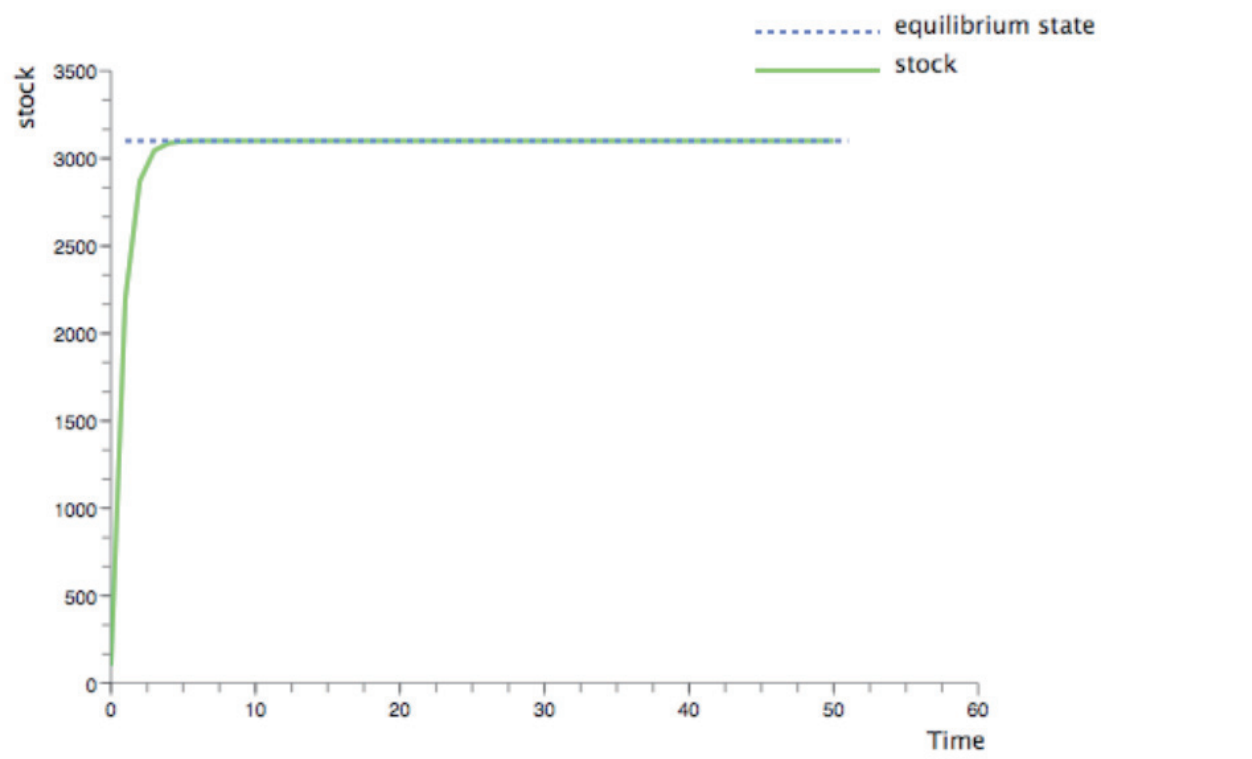

Figure 1. Evolution of the stock fish population in the stage class 1 with the feedback $E(k)=E^{*}+v(X(k))$ 


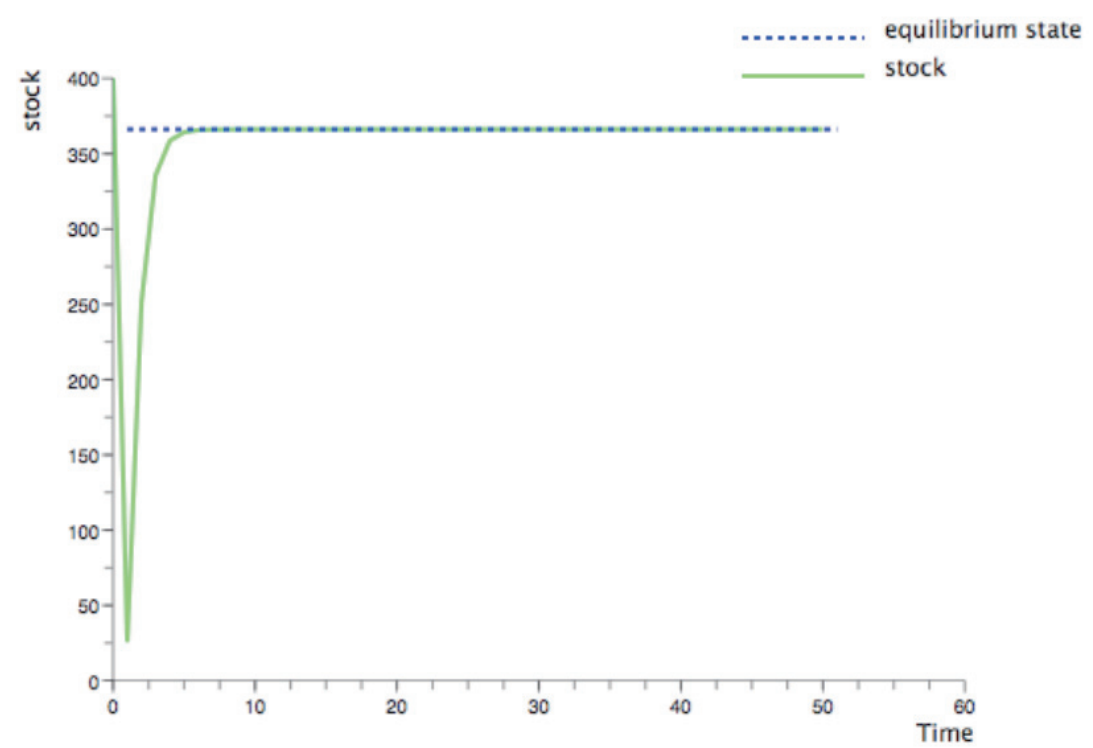

Figure 2. Evolution of the stock fish population in the stage class 2 with the feedback $E(k)=E^{*}+v(X(k))$

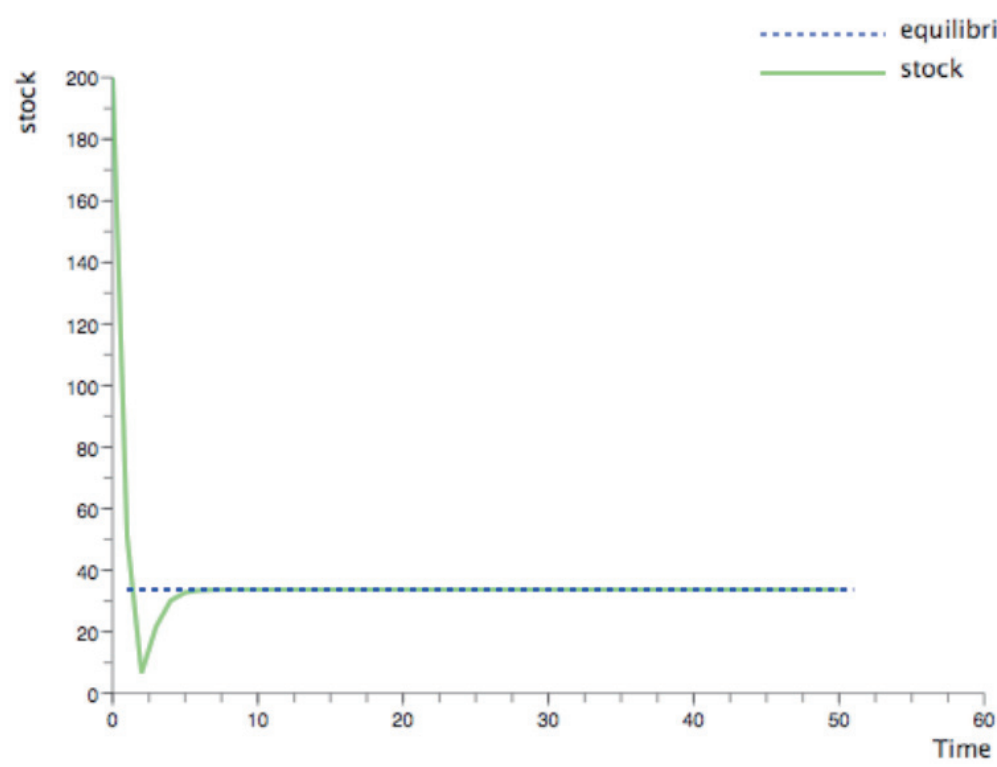

Figure 3. Evolution of the stock fish population in the stage class 3 with the feedback $E(k)=E^{*}+v(X(k))$

\section{References}

Bensoubaya, M., Ferfera, A., \& Iggidr, A. (1995a). Stabilisation de systèmes non linéaires discrets. Comptes Rendus de l'Académie des Sciences Paris, Série I, 321, 371-374.

Bensoubaya, M., Ferfera, A., \& Iggidr, A. (1995b). On the stabilization of continuous and discrete time nonlinear systems. In The 2nd Marrakesh International Conference on Differential Equations.

Beverton, R. J. H., \& Holt, S. J. (1957). On the dynamics of exploited fish populations. Chapman \& Hall.

Damania, S. P., Phillips, K. W., Henson, S. M., \& Hayward, J. L. (2005). Habitat patch occupancy dynamics of glaucous-winged gulls (Larus glaucescens) II: a continuous-time model. Natural Resource Modeling, 18, 469-499. http://dx.doi.org/10.1111/j.1939-7445.2005.tb00168.x

Doubleday, W. (1975). Harvesting in matrix population models. Biometrics, 31, 189-200. http://dx.doi.org/10.2307/2529719

Elaydi, S. N. (1996). An Introduction to Difference Equations. New York: Springer-Verlag. 
El Bagdouri, M., Iggidr, A., Ouahbi, A., \& Vivalda, J. C. (2000). On the regulation of the state of a harvested fish population. Proc. the 2000 American Control Conference, 3336-3337.

Getz, W. M., \& Haight, R. G. (1989). Population harvesting. Demographic models of fish, forest, and animal resources. Monographs in Population Biology, 27. Princeton, NJ: Princeton University Press.

Gouze, J. L., Rapaport, A., \& Hadj-Sadok, M. Z. (2000). Interval observers for uncertain biological systems. J. Ecol. Modell., 133(1), 45-56.

Guiro, A., Iggidr, A., \& Ngom, D. (2008). Interval Numerical Observer: Application to a discrete time nonlinear fish model. In Proc. African Conference on Research in Computer and Applied Mathematics, 69-76.

Guiro, A., Iggidr, A., \& Ngom, D. (2011). On the Stock Estimation for a Harvested Fish Population. Bull Math Biol, 74(1), 116-142. http://dx.doi.org/10.1007/s11538-011-9667-z

Henson, S. M., Hayward, J. L., \& Damania, S. P. (2005). Identifying environmental determinants of diurnal distribution in marine birds and mammals. Bulletin of Mathematical Biology, 68, 467-482. http://dx.doi.org/10.1007/s11538-005-9009-0

Hurt, J. (1967). Some Stability theorems for ordinary difference equations. IMA SIAM. J. Numer. Anal., 4, 582596. http://dx.doi.org/10.1137/0704053

Iggidr, A. (2006). Controllability, observability and stability of mathematical models. in Mathematical Models. In Encyclopedia of Life Support Systems (EOLSS). In J. A. Filar (Ed.), Developed under the auspices of the UNESCO. Oxford, UK: Eolss Publishers. Retrieved from http://www.eolss.net

Jurdjevic, V., \& Quinn, J. P. (1978). Controllability and stability. Journal of Differential Equations, 28, $381-389$. http://dx.doi.org/10.1016/0022-0396(78)90135-3

Kalman, R. E., \& Bertram, J. E. (1960). Control system analysis and design via the second method of lyapounov II discrete systems. Trans ASME Ser D. J. Basic Engineering, 82, 394-400. http://dx.doi.org/10.1115/1.3662605

Lasalle, J. P. L. (1986). The stability and control of discret processes. New York: Springer-Verlag. http://dx.doi.org/10.1007/978-1-4612-1076-4

Lin, W. (1996). Further results on global stabilization of discrete nonlinear systems. Systems \& Control Letters, 29, 51-59. http://dx.doi.org/10.1016/0167-6911(96)00037-0

Magal, P., \& Pelletier, D. (1997). A fixed point theorem with application to a model of population dynamics. $J$. Difference Equ. Appl., 3(1), 65-87. http://dx.doi.org/10.1080/10236199708808085

Ngom, D., Iggidr, A., Guiro, A., \& Ouahbi, A. (2008). An Observer for a nonlinear age-structured model of a harvested fish population. Mathematical Biosciences and Engineering, 5(2), 337-354. http://dx.doi.org/10.3934/mbe.2008.5.337

Ouahbi, A. (2002). Observation et Contrôle de modèles non-linéaires de populations marines exploitées (Ph.D thesis, University of Marrakech, Morocco).

Ricker, W. E. (1954). Stock and recruitment. J. Fish. Res. Board Can., 11, 559-623. http://dx.doi.org/10.1139/f54-039

Shepherd, J. G. (1982). A family of general production curves for exploited populations. Mathematical Biosciences, 59, 77-93. http://dx.doi.org/10.1016/0025-5564(82)90110-9

Sontag, E. D. (1998). Mathematical control theory. Deterministic finite-dimensional systems. Texts in Applied Mathematics, 6. New York: Springer-Verlag. 